\title{
Neural Underpinnings of the Identifiable Victim Effect: Affect Shifts Preferences for Giving
}

\author{
Alexander Genevsky, ${ }^{1}$ Daniel Västfjäll, ${ }^{2,3}$ Paul Slovic, ${ }^{3,4}$ and Brian Knutson ${ }^{1}$ \\ ${ }^{1}$ Psychology and Neuroscience, Stanford University, Stanford, California 94305-2130, 2Psychology, Linkoping University, 58183 Linkoping, Sweden, \\ ${ }^{3}$ Decision Research, Eugene, Oregon 97401, and ${ }^{4}$ University of Oregon, Eugene, Oregon 97403
}

\begin{abstract}
The "identifiable victim effect" refers to peoples' tendency to preferentially give to identified versus anonymous victims of misfortune, and has been proposed to partly depend on affect. By soliciting charitable donations from human subjects during behavioral and neural (i.e., functional magnetic resonance imaging) experiments, we sought to determine whether and how affect might promote the identifiable victim effect. Behaviorally, subjects gave more to orphans depicted by photographs versus silhouettes, and their shift in preferences was mediated by photograph-induced feelings of positive arousal, but not negative arousal. Neurally, while photographs versus silhouettes elicited activity in widespread circuits associated with facial and affective processing, only nucleus accumbens activity predicted and could statistically account for increased donations. Together, these findings suggest that presenting evaluable identifiable information can recruit positive arousal, which then promotes giving. We propose that affect elicited by identifiable stimuli can compel people to give more to strangers, even despite costs to the self.
\end{abstract}

\section{Introduction}

Contrary to the dictates of pure self-interest, theorists have argued that individuals can be moved to help vivid and identifiable others (Fetherstonhaugh et al., 1997; Jenni and Loewenstein, 1997; Kogut and Ritov, 2005a; Slovic, 2007). This preference for giving to single vivid individuals over less identifiable others has been called the "identifiable victim effect." The identifiable victim effect resists explanation by normative economic models, since identifiable stimuli add no objective value or relevant information. While research has established the behavioral robustness of the identifiable victim effect (Andreoni and Petrie, 2004; Kogut and Ritov, 2013), the psychological and neural mechanisms underpinning this effect remain unclear.

Researchers have speculated that affect may play a role in the identifiable victim effect (Kogut and Ritov, 2005a), but have not established exactly how or in which direction. On the one hand, a number of research findings suggest that positive affect (i.e., a "warm glow") evoked by anticipation of giving can promote empathic and charitable behavior (Andreoni, 1990, 1995; Harbaugh et al., 2007). On the other hand, a distinct set of findings implies

\footnotetext{
Received June 3, 2013; revised Sept. 23, 2013; accepted Sept. 26, 2013.

Author contributions: A.G., D.V., P.S., and B.K. designed research; A.G. performed research; B.K. contributed unpublished reagents/analytic tools; A.G. and B.K. analyzed data; A.G., D.V., P.S., and B.K. wrote the paper.

This research was supported by a grant from the Stanford Center for Compassion and Altruism Research and Education (CCARE) to B.K., and A.G. was supported by National Institutes of Health Training Grant 5T32MH02000615.P.S. and D.V. acknowledge support from National Science Foundation Grants SES-1024808 and SES-1227729. We thank James Andreoni, Jamil Zaki, and three anonymous reviewers for helpful comments on previous drafts of this manuscript.

The authors declare no competing financial interests.

This article is freely available online through the J Neurosci Author Open Choice option.

Correspondence should be addressed to Alexander Genevsky, Department of Psychology, Stanford University, Jordan Hall, 420 Serra Mall, Stanford, CA 94305-2130. E-mail: genevsky@stanford.edu.

DOI:10.1523/JNEUROSCI.2348-13.2013

Copyright $\odot 2013$ the authors $\quad 0270-6474 / 13 / 3317188-09 \$ 15.00 / 0$
}

that negative affect evoked by empathic pain can increase prosocial behavior (Small and Verrochi, 2009; Hein et al., 2010; Masten et al., 2011). Thus, it is not clear whether positive aroused feelings (e.g., excitement, enthusiasm), negative aroused feelings (e.g., guilt, anxiety), or some combination of these factors increases giving. Further, neural mechanisms that underlie the identifiable victim effect have not been characterized. Existing studies of the identifiable victim effect have manipulated potential causes between subjects (e.g., with different vignettes) rather than within subjects, which might facilitate study with neuroimaging techniques (Rosen et al., 1998).

Although neuroimaging studies have not yet focused on the identifiable victim effect, some work has explored neural antecedents of charitable giving. Numerous neuroimaging studies now indicate that anticipation of monetary and other gains increase nucleus accumbens (NAcc) activity as well as associated positive aroused affect, while anticipation of monetary and other losses increase anterior insula activity as well as associated negative aroused or generally aroused affect (Knutson and Greer, 2008). Consistent with the notion that anticipatory affect might promote giving, researchers have found that individual differences in neural activity associated with positive arousal (i.e., in regions including the NAcc) during forced donations predict later voluntary giving (Moll et al., 2006; Harbaugh et al., 2007; Hare et al., 2010). These findings might suggest that individual differences in positive affect associated with giving (e.g., a warm glow) promote charitable donation (Andreoni, 1990). No neuroimaging studies, however, have independently manipulated affect within individuals to examine whether changes in affect could shift individuals' preferences for giving.

In the present research, we first sought to behaviorally determine whether and how identifiable information might shift preferences for giving within subjects. We then sought to neurally 
A

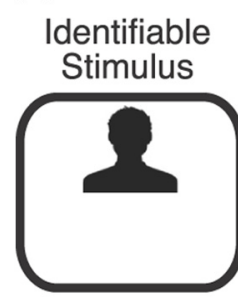

$4 \mathrm{sec}$

B

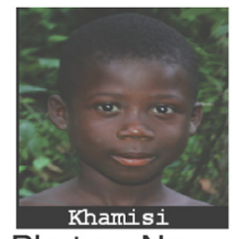

Photo + Name

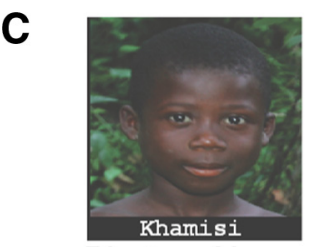

Photo + Name
Requested

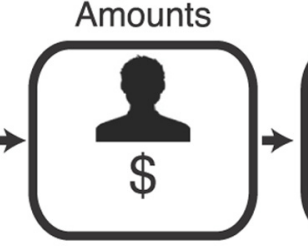

$2 \mathrm{sec}$

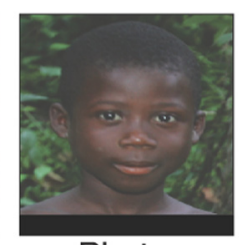

Photo

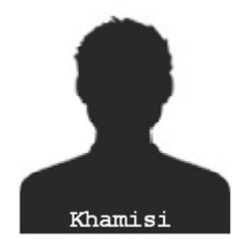

Name
Choice

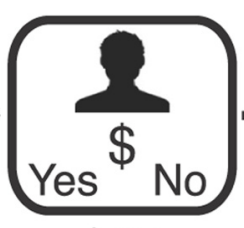

$4 \mathrm{sec}$

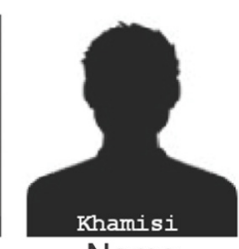

Name
Fixation

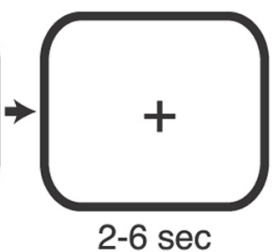

2-6 sec

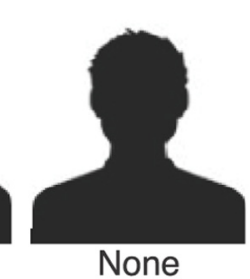

None
Figure 1. Experimental designs. $\boldsymbol{A}$, Trial structure included screens presenting identifiable information ( $4 \mathrm{~s})$, requested donation amount ( $2 \mathrm{~s}$ ), choice ( $4 \mathrm{~s})$, and variable fixation interval ( $2-6 \mathrm{~s}$; average trial length is $14 \mathrm{~s})$. $\boldsymbol{B}$, Identifiable information conditions in the behavioral experiment (i.e., Photo + Name, Photo, Name, and No Info). C, Identifiable information conditions in the neuroimaging experiment (i.e., Photo + Name and Name).

verify whether identifiable information would recruit specific brain circuits associated with affect, and whether activity in these circuits would promote giving. Based on an anticipatory affect account (Knutson and Greer, 2008) we predicted that identifiable information that increases positive arousal and associated NAcc activity would promote giving, while remaining open to the alternate possibilities that negative arousal and correlated anterior insula activity, or activity in other relevant (e.g., sensory or motor) circuits might promote giving.

\section{Materials and Methods}

\section{Behavioral study}

Subjects. Fourteen undergraduate and graduate students at Stanford University participated in the behavioral study. Three of these subjects $(21 \%)$ either always $(n=2)$ or never $(n=1)$ chose to donate, providing insufficient data to model, so were excluded from further analysis, leaving a total of 11 subjects ( six female, mean age $=22$ years). Subjects were screened for psychotropic drugs, substance abuse in the past month, and history of neurological disorders before collecting informed consent. Subjects received an endowment minus their contribution to an African orphan on a randomly selected trial (see below), as well as $\$ 15.00$ per hour for their time.

Procedure. To elicit personally consequential donations, subjects received a $\$ 15.00$ endowment immediately before the donation task, and physically placed the money in their pocket. Subjects were assured that this additional money was theirs to keep, but also that any portion they decided to donate on a randomly selected trial would be collected and donated to an African orphanage after the experiment. The experimenter informed subjects that they had established a partnership with a children's refugee orphanage in the Darfur region of Sudan, and that subjects would be asked to donate a portion of their $\$ 15$ endowment to specific children in each trial of the experiment. Subjects were told that approximately 60 children lived at the orphanage, but that variable amounts of information (i.e., photographs, names) were available for each and would be presented during each trial. Finally, subjects were informed that only one of the trials would be randomly selected to "count for real" at the end of the task, so they should treat each decision as independent, and should not spread their donation evenly over trials or save money for later trials.

Donation task. During each trial, subjects first viewed a screen with information depicting the potential recipient of their donation (4 s) followed by the amount of the donation requested (e.g., \$5) for that recipient (2 s). Subjects were then asked to indicate whether or not they would donate the requested amount using "Yes" and "No" options appearing in counterbalanced positions (left vs right) at the bottom of the screen, by pressing a spatially corresponding button on a button box (4 s). Once their response was registered, feedback indicating their choice with a colored border was displayed onscreen until the end of the choice period. Finally, subjects fixated on a cross (variable 2-6s) before the next trial began (Fig. $1 A)$. Overall, average trial duration (including intertrial interval) was $14 \mathrm{~s}$ (range $=12-16 \mathrm{~s}$ ). Subjects encountered 15 trials in each of four conditions ("Photo + Name," "Photo," "Name," and "No Information"; Fig. 1B), equally distributed along the range of requested amounts (\$1-12), which was subsequently divided into three ordinal levels for analyses $($ Low $=\$ 1-4 ;$ Med $=\$ 5-8$; High $=$ \$9-12). Photographs purportedly depicted the faces of actual children at the orphanage, and to maintain the impression that distinct individuals were being considered during each trial, silhouettes (rather than scrambled faces) served as control stimuli for photographs.

Affect. Following the Donation Task, subjects completed an abbreviated affect rating task, in which they rated their affective reactions to scenarios that were representative of each condition on two scales indexing valence (positive-negative) and arousal (highly arousing-not arousing). Verbal and written instructions delivered by the experimenter explicitly described the nature of these two independent (seven point) scales, and detailed examples were provided (Knutson et al., 2008). Rating trials then displayed screenshots mimicking each condition of the Donation Task, with information about a potential recipient and a requested amount. Subjects then entered valence and arousal ratings using the number keys on a keypad, based upon how they felt during the previous task "when asked to give this amount of money, given the information provided about the potential recipient." To avoid rating biases related to memories of previous choices, the representative scenarios involved novel faces and names. For ratings, donation requests were binned into Low (\$1-4), Medium (\$5-8), and High (\$9-12) amounts. Since aroused affect theoretically potentiates motivated approach or avoidance, and empirically correlates most robustly with activity in targeted neural circuits (Knutson and Greer, 2008), valence and arousal ratings were transformed into positive arousal (PA) and negative arousal (NA) scores. These transformations involved mean-deviating valence and arousal scores within subject and item and then applying a 45 degree rotation to the transformed scores [i.e., $P A=A r / \sqrt{2}+V a / \sqrt{2}$; $N A=A r / \sqrt{2}-V a / \sqrt{2}$ ] (Watson et al., 1999; Knutson et al., 2005). Positive arousal and negative arousal scores were then averaged by condition for analyses.

\section{Neuroimaging study}

Subjects. Thirty healthy right-handed adults participated. Eight $(26 \%)$ of these subjects either always $(n=4)$ or never $(n=4)$ chose to donate and so did not provide sufficient data to model, leading to exclusion from 
further analyses, and leaving a total of 22 subjects ( 14 females; age range $18-26)$ for the final analysis. Along with the typical magnetic resonance exclusions (e.g., metal in the body), subjects were screened for psychotropic drug use and substance abuse in the past month as well as for a history of neurological disorders before collecting informed consent. Subjects were paid $\$ 15.00$ per hour for participating and also received an endowment of $\$ 15.00$ to use in the donation task before scanning.

Donation task. As in the behavioral study, subjects were asked in successive trials whether they would choose to donate different specific amounts of their endowment to orphans. During each trial, subjects first viewed a screen with information about the potential recipient of their donation ( $4 \mathrm{~s}$ ) followed by the amount of the donation requested (e.g., $\$ 5.00)$ for that particular child ( $2 \mathrm{~s})$. Subjects were then asked to indicate whether or not they would donate the requested amount using Yes and No options appearing in counterbalanced positions (left vs right) at the bottom of the screen ( $4 \mathrm{~s})$. Finally, subjects fixated on a cross (variable $2-6 \mathrm{~s}$ ) before the beginning of the next trial (Fig. 1A). Note that while intertrial intervals were temporally jittered, events within a trial were not, to facilitate extraction of precisely timed peak activity in response to the presentation of new information (e.g., photo or silhouette, amount requested) for subsequent trial-based prediction and mediation analyses (Knutson et al., 2007; Grosenick et al., 2013). Subjects encountered 30 trials in each of two conditions ("Photo," or "'Silhouette"; Fig. 1C), equally distributed along the range of requested amounts (\$1-12), which was subsequently divided into three ordinal levels for analyses (Low $=$ $\$ 1-4 ;$ Med $=\$ 5-8 ;$ High $=\$ 9-12$ ). Following the session, one trial was selected at random to count for real. If the subject had agreed to donate, the amount was removed from their payment. Otherwise, they retained their full endowment. As in the behavioral study, subjects participated in a reduced condition affect rating task immediately following the scanned donation task.

Functional magnetic resonance imaging acquisition and analysis. Images were acquired with a 3.0 T General Electric magnetic resonance imaging scanner using a 32 channel head coil. Forty-six 2.9-mm-thick slices (inplane resolution $2.9 \mathrm{~mm}$ isotropic, no gap, interleaved acquisition) extended axially from the mid-pons to the crown of the skull, providing whole-brain coverage and good spatial resolution of subcortical regions of interest (e.g., midbrain, NAcc, orbitofrontal cortex). Whole-brain functional scans were acquired with a $\mathrm{T} 2^{*}$-weighted gradient echo pulse sequence $\left(\mathrm{TR}=2 \mathrm{~s}, \mathrm{TE}=24 \mathrm{~ms}\right.$, flip $\left.=77^{\circ}\right)$. High-resolution structural scans were acquired with a T1-weighted pulse sequence $(\mathrm{TR}=7.2 \mathrm{~ms}$, $\mathrm{TE}=2.8 \mathrm{~ms}$, flip $=12^{\circ}$ ) after functional scans, to facilitate their localization and coregistration.

Whole-brain analyses were conducted using Analysis of Functional Neural Images (AFNI) software (Cox, 1996). For preprocessing, voxel time series were sinc interpolated to correct for nonsimultaneous slice acquisition within each volume, concatenated across runs, corrected for motion, slightly spatially smoothed to minimize effects of anatomical variability (full-width at half-maximum $=4 \mathrm{~mm}$ ), high-pass filtered (admitting frequencies with period $<90 \mathrm{~s}$ ), and normalized to percentage signal change with respect to each voxel's average over the entire task. Visual inspection of motion correction estimates confirmed that no subject's head moved $>2.0 \mathrm{~mm}$ in any dimension from one volume acquisition to the next.

For whole-brain analyses, regression models included eight regressors of no interest, six of which indexed residual motion, and two of which indexed CSF and white matter intensity (Chang and Glover, 2009). In a first model, regressors of interest orthogonally contrasted the following: (1) photograph versus silhouette presentation, (2) low versus high amount requested, and (3) their interaction-all during simultaneous presentation of photographs and amount requested (which occurred $2 \mathrm{~s}$, or one volume acquisition, before the choice prompt onset). In a second model, the single regressor of interest contrasted anticipation of choosing to donate or not during simultaneous presentation of photographs and amount requested (which occurred before the choice prompt onset). Before inclusion in the models, regressors of interest were convolved with a single gamma-variate function that modeled a canonical hemodynamic response (Cohen, 1997). Maps of $t$ statistics for regressors of interest were transformed into $Z$ scores, coregistered with structural maps, spatially normalized by warping to Talairach space, and resampled as 2 $\mathrm{mm}$ cubic voxels. Group maps were initially voxelwise thresholded (at $p<0.005$ ) and then cluster thresholded (cluster size $>9$ contiguous 3 $\mathrm{mm}$ cubic voxels) to yield a corrected threshold for detecting wholebrain activation ( $p<0.05$ corrected, derived with 15,000 Monte Carlo iterations using AFNI program 3dClustSim).

Targeted analyses were conducted by specifying volumes of interest in regions associated with anticipatory affect (Knutson and Greer, 2008) and charitable giving (Harbaugh et al., 2007) in previous research. These regions also showed significant activation in the independent face versus silhouette contrast in the present study. Specifically, spherical volumes of interest ( $8 \mathrm{~mm}$ diameter) were placed at bilateral foci in the NAcc (Talairach coordinates: $\pm 10,12,-2$ ), and anterior insula (Talairach coordinates, $\pm 34,24,-4)$. Similar volumes of interest were also placed on bilateral amygdala foci (Talairach coordinates, $\pm 24,-5,-15)$ and bilateral medial prefrontal cortical (MPFC) foci (Talairach coordinates, \pm 4 , $45,0)$, as control regions in which we predicted that although faces versus silhouettes might increase activity, activity would not predict choice. Activity (percentage signal change) was averaged within each volume of interest, averaged across bilateral volumes of interest, and then extracted to derive activation time courses.

For prediction analyses, percentage signal change during concurrent presentation of photographs and requested amount $2 \mathrm{~s}$ before the choice prompt onset (i.e., the third volume acquisition in the trial) lagged by $4 \mathrm{~s}$ (to account for the hemodynamic response) were submitted to logistic regression models that used neural activity to predict choices to donate or not on a trial-to-trial basis (Knutson et al., 2007). Models included positive and negative arousal, and peak neural activity extracted from bilateral NAcc, bilateral anterior insula, bilateral amygdala, and bilateral MPFC volumes of interest. To establish generalizability across subjects, subjects were modeled as random effects, and neural models were further subjected to leave-one-subject-out cross-validation. To determine whether affect and brain activity mediated the influence of identifiable stimuli on choice, bootstrapped mediation analyses were conducted (Sobel, 1982; Mackinnon and Lockwood, 2004; Preacher and Hayes, 2008).

\section{Results}

\section{Behavioral study}

Choice

In a behavioral study $(n=11)$, we predicted that affectively compelling identifiable information would influence decisions to donate. Trial-based choices to donate or not were analyzed using a hierarchical logistic regression model that included fixed effects of photograph (vs silhouette), name (vs no name), and donation amount (in U.S. dollars), as well as random effects of subjects. Significance testing was performed by comparing two models (via log likelihood), differing only by the exclusion of a factor of interest, using a $\chi^{2}$ test with one degree of freedom (Jaeger, 2008). This model revealed a significant effect of photograph (vs silhouette; $\left.\chi^{2}(1)=25.96, p<0.001\right)$ on donations, such that subjects donated more often on trials with photographs (i.e., Photo + Name and Photo trials; 64\%) than those with silhouettes (i.e., Name and None trials; 27\%; Fig. 2A). In contrast, there were no significant effects of name (vs no name; $\chi^{2}(1)=0.74, p=0.389$ ) or the interaction of photograph and name $\left(\chi^{2}(1)=1.84, p=\right.$ $0.175)$ on donations. There was also a significant effect of requested amount on donations $\left(\chi^{2}(1)=296.54, p<0.001\right)$, such that low requested amounts increased donations. There were no significant interactions between photograph $\left(\chi^{2}(1)=2.12, p=\right.$ $0.144)$ or name $\left(\chi^{2}(1)=0.14, p=0.708\right)$ and requested amount. Thus, photographs increased donations-possibly by eliciting affect.

\section{Affect}

Choice results suggested that photographs, but not names, might influence affect. Conditional affect ratings in the affect rating task 
A

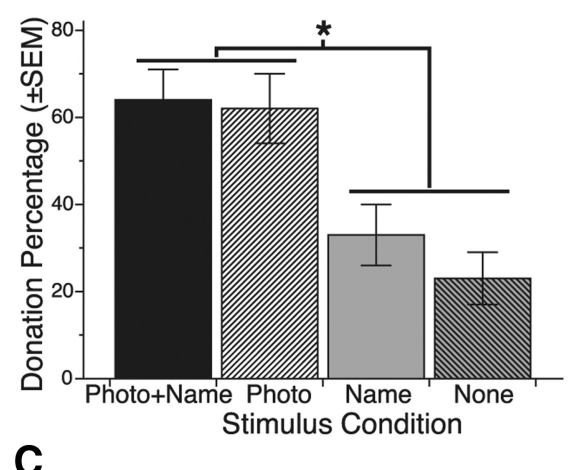

C

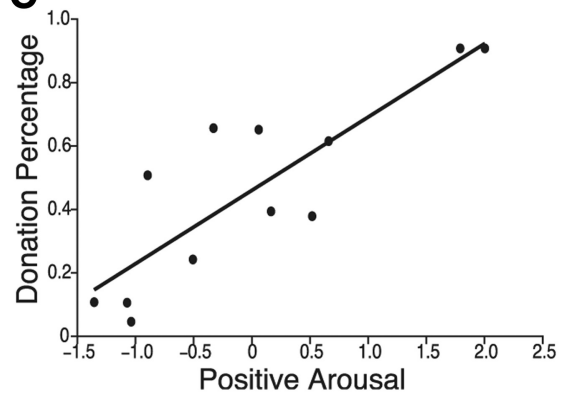

B

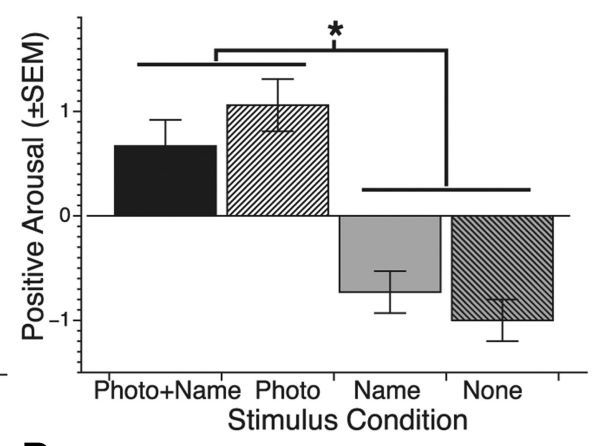

D

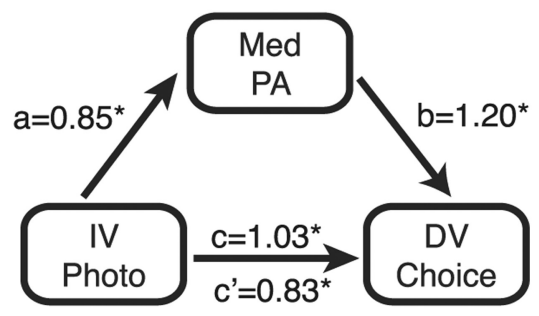

Figure 2. Identifiable stimuli behaviorally promote giving by eliciting positive arousal. $\boldsymbol{A}$, Significantly greater probability of giving for trials with photographs than silhouettes, but no effect of name. $\boldsymbol{B}$, Significantly higher positive arousal elicited by trials with photos than silhouettes, but no effect of name. $\boldsymbol{C}$, Significant positive correlation $(r=0.71, p<0.01)$ of positive arousal ratings with probability of giving across conditions (data points represent average positive arousal and percentage donation by condition; i.e., 4 stimulus configurations $\times 3$ requested amounts). $D$, PA statistically mediates the relationship between presentation of photographs versus silhouettes and percentage donations (Choice). The indirect path from photograph to positive arousal to donation decision was significant $(p<0.001, Z=3.29$; index of mediation $=0.271,95 \% \mathrm{Cl}=[0.228,0.312])$. The direct path coefficient of photograph to donation decision decreased in the model after including positive arousal as a mediator (from 1.03 to $0.83 ; Z=3.29, p<0.001)$.

were analyzed using a 2 (photograph vs silhouette) $\times 2$ (name vs no name) $\times 3$ (low, medium, high requested amount) ANOVA. This analysis revealed a main effect of photograph $\left(F_{(1,10)}=\right.$ $27.74, p<0.001$ ) on positive arousal, such that photographs (i.e., Photo + Name and Photo trials) elicited greater positive arousal than silhouettes (Fig. 2B). There was, however, no significant main effect of name (i.e., Photo + Name and Name; $F_{(1,10)}=0.82$, $p=0.386)$ or face by name interaction $\left(F_{(1,10)}=1.44, p=0.260\right)$ on positive arousal. Unlike positive arousal, presentation of photographs $\left(F_{(1,10)}=0.82, p=0.389\right)$ and names $\left(F_{(1,10)}=0.22, p=\right.$ $0.650)$ had no significant effects on negative arousal. These findings indicate that photographs influenced positive arousal but not negative arousal. Amount requested influenced both positive arousal $\left(F_{(2,20)}=13.38, p<0.001\right)$ and negative arousal $\left(F_{(2,20)}=7.29, p=\right.$ $0.004)$, such that low versus high requested amounts increased positive arousal $\left(t_{(10)}=4.27, p=0.002\right)$ but decreased negative arousal $\left(t_{(10)}=-3.00, p=0.013\right)$. Photographs and requested amount also significantly interacted in their influence on positive arousal $\left(F_{(6,54)}=3.63, p=0.004\right)$ but not negative arousal $\left(F_{(6,54)}=1.04\right.$, $p=0.410)$.

\section{Influence of affect on choice}

We further predicted that positive arousal elicited by identifiable information might mediate the influence of photographs on donations. Affect ratings were collected after the donation task on an abbreviated representative sample of trials from each condition to maximize task realism, minimize affective priming of choice, and reduce interruptions during the process of decision making. Initial bivariate correlation analyses indicated that con- ditions that elicited more positive arousal also elicited higher percentage donations $(r=0.71, p=0.014$; Fig. $2 C)$, while conditions that elicited higher negative arousal did not (and if anything, elicited lower percentage donations; $r=-0.46$, $p=0.155)$.

Bootstrapped mediation analyses (Sobel, 1982; Mackinnon and Lockwood, 2004; Preacher and Hayes, 2008) were then conducted to examine whether conditional affect ratings could mediate the trial-based influence of photographs on choices to donate (Fig. 2D). A distribution of the indirect (mediated) effect, calculated as the product of paths from the independent variable to the mediator and from the mediator to the dependent variable (i.e., $\mathrm{a}^{\star} \mathrm{b}$ ), was derived over 1000 iterations of data sampled with replacement. Reported $p$ values were calculated as the proportion of this distribution that exceeded a null value of 0 . Results revealed a significant indirect path from photograph to positive arousal to choices to donate $(p<0.001, Z=3.29$; index of mediation $=0.271,95 \%$ confidence interval $(C I)=[0.228,0.312])$. Further, the direct path coefficient from photograph to choices to donate decreased in the model after including positive arousal as a mediator (i.e., from 1.03 to $0.83 ; Z=3.29, p<$ $0.001)$. Together, these findings indicate that the influence of photographs on choices to donate was partially mediated

by positive arousal.

Findings from this behavioral study suggested that identifiable information primarily increased donations by eliciting positive arousal. Since activity in specific brain circuits (i.e., the NAcc) has been associated with positive arousal (Knutson and Greer, 2008), these findings implicate a neural target through which identifiable information might promote giving. Further, the size of the within subjects effects supported a power analysis of the number of subjects needed to replicate these behavioral effects.

\section{Neuroimaging study}

In a neuroimaging study $(n=22)$ we sought to replicate and extend behavioral findings by probing neural activity before subjects chose to donate money to orphans. Since photographs but not names elicited anticipatory affect and increased donations, only the photograph versus silhouette manipulation was included (Fig. 1C). Based on the robust within-subject effect of photographs on donations observed in the behavioral study (i.e., $Z=4.62$ for the photograph vs silhouette effect in the hierarchical logistic regression model), power analyses indicated that at least seven subjects would be required to replicate the behavioral effect (power $=0.80, p<0.05$, two-tailed).

Choice

As in the behavioral study, we predicted that photographs and requested amount would influence choices to donate. Trialbased choices to donate or not were analyzed using a hierarchical 
logistic regression model that included fixed effects of photograph (vs silhouette) and donation amount, as well as random effects of subjects. Significance testing was performed by comparing two models (via log likelihood statistic), differing only by the exclusion of a factor of interest, using a $\chi^{2}$ test with one degree of freedom (Jaeger, 2008). As in the behavioral study, subjects donated more on trials with photographs (mean: 59.4\%, SEM: 5.03) than on those with silhouettes (mean: 40.9\%, SEM: $4.70-$ an average difference of $\$ 2.22 ; \chi^{2}(1)=12.71, p<0.001$; see Fig. $4 A$ ). Further, lower requested donation amount increased donations $\left(\chi^{2}(1)=537.94, p<0.001\right)$. There were no significant interactions between photographs and requested amount on donations $\left(\chi^{2}(1)=0.16, p<0.693\right)$. Auxiliary group contrasts indicated no significant gender differences in percentage choices to donate $\left(t_{(20)}=1.83, p=0.082\right)$, either in the photograph trials $\left(t_{(19)}=1.68, p=0.109\right)$, or in the silhouette trials $\left(t_{(20)}=1.60, p=0.125\right)$.

\section{Affect}

As with choice, we predicted that photographs and requested amount would influence affect. Conditional affect ratings were acquired after scanning during an affective rating task involving representative trials, as in the behavioral study. Conditional affect ratings were analyzed using a 2 (photograph vs silhouette) $\times 3$ (low, medium, or high requested amounts) within-subject mixed effects ANOVA. A main effect of photograph versus silhouette on affect ratings indicated that photographs increased self-reported positive arousal $\left(F_{(1,21)}=52.89, p<0.001\right)$, as well as negative arousal $\left(F_{(1,21)}=11.71, p=0.003\right)$. Requested amount also influenced positive arousal $\left(F_{(2,42)}=4.31, p=0.019\right)$, such that Low versus High $\left(t_{(21)}=2.10, p=0.048\right)$ requested amounts increased positive arousal. Requested amount additionally influenced negative arousal $\left(F_{(2,42)}=5.40, p=0.008\right)$, such that Low versus High $\left(t_{(21)}=-2.61, p=0.016\right)$ requested amounts decreased negative arousal. Photographs and requested amount did not significantly interact in their influence on positive arousal $\left(F_{(2,42)}=1.00, p=0.376\right)$ or negative arousal $\left(F_{(2,42)}=1.17, p=0.320\right)$.

\section{Influence of affect on choice}

Consistent with findings from the behavioral study, we predicted that positive arousal but not negative arousal would significantly mediate the influence of photographs on donations. Initial bivariate correlation analyses again indicated that conditions that elicited more positive arousal also elicited higher percentage donations $(r=0.44, p=0.040)$, but conditions that elicited higher negative arousal did not $(r=-0.16, p=0.477)$. Bootstrapped mediation analysis $(n=1000)$ demonstrated a significant indirect effect of the path from photograph to positive arousal to choices to donate $(p<0.001, Z=3.29$; index of mediation $=$ $2.50,95 \% \mathrm{CI}=[2.08,3.86])$, and a decrease in the direct path coefficient from photograph to choices to donate after including positive arousal as a mediator (from 0.87 to $0.56 ; Z=3.29, p<$ 0.001 ), indicating partial mediation by positive arousal.

\section{Whole-brain activity}

In the first model, we predicted that photographs versus silhouettes would activate regions commonly associated with face processing (e.g., visual ventral stream, fusiform face area, and amygdala; Kanwisher et al., 1997), as well as regions implicated in anticipatory affect (e.g., NAcc and anterior insula; Knutson and Greer, 2008). Whole-brain analyses revealed that the photograph versus silhouette contrast elicited increased activity in regions implicated in visual and face processing (including the ventral
Table 1. Whole-brain analyses contrasting exposure to photographs versus silhouettes, requests for low versus high donations, and their interaction

\begin{tabular}{|c|c|c|c|c|c|c|}
\hline & Region & $x$ & $y$ & $z$ & Peak Z & Voxels \\
\hline \multirow{19}{*}{$\begin{array}{l}\text { Photo versus } \\
\text { silhouette }\end{array}$} & R visual cortex & 16 & -83 & -6 & 6.04 & 1621 \\
\hline & L visual cortex & -13 & -83 & -9 & 5.74 & 1159 \\
\hline & L amygdala & -13 & -5 & -9 & 5.22 & 245 \\
\hline & $\begin{array}{l}\text { R superior temporal } \\
\text { gyrus }\end{array}$ & 59 & -28 & 8 & -3.68 & 71 \\
\hline & R MPFC & 2 & 44 & -3 & 3.72 & 38 \\
\hline & L posterior cingulate & -4 & -46 & 20 & 3.86 & 32 \\
\hline & R inferior parietal lobule & 48 & -51 & 46 & -3.43 & 26 \\
\hline & L anterior insula & -25 & 15 & -9 & 3.90 & 24 \\
\hline & Left thalamus & -2 & -16 & 8 & 3.61 & 24 \\
\hline & $R$ anterior insula & 30 & 21 & -9 & 3.61 & 23 \\
\hline & L anterior cingulate & -4 & 39 & 12 & 3.58 & 22 \\
\hline & R cerebellar tonsil & 1 & -48 & -38 & 3.65 & 20 \\
\hline & R medial frontal gyrus & 7 & 47 & 35 & 3.83 & 18 \\
\hline & R medial frontal gyrus & 7 & 4 & 61 & 3.55 & 18 \\
\hline & $\begin{array}{l}\text { R superior temporal } \\
\text { gyrus }\end{array}$ & 54 & -28 & 14 & -3.35 & 17 \\
\hline & R inferior frontal gyrus & 45 & 36 & 3 & -3.33 & 16 \\
\hline & L precentral gyrus & -54 & -2 & 14 & -3.73 & 14 \\
\hline & L inferior parietal lobule & -60 & -40 & 29 & -3.30 & 12 \\
\hline & R NACC & 4 & 12 & -3 & 4.17 & 11 \\
\hline \multirow{13}{*}{$\begin{array}{l}\text { Low versus high } \\
\text { requested } \\
\text { amount }\end{array}$} & L middle occipital gyrus & -30 & -74 & 14 & 4.43 & 216 \\
\hline & R precentral gyrus & 30 & -11 & 67 & 3.78 & 27 \\
\hline & R cerebellar culmen & -1 & 63 & 3 & 3.48 & 27 \\
\hline & R middle occipital gyrus & 28 & -74 & 14 & 3.99 & 24 \\
\hline & $\begin{array}{l}\text { R superior temporal } \\
\text { gyrus }\end{array}$ & 62 & -34 & 14 & 3.98 & 24 \\
\hline & R precuneus & 25 & -46 & 55 & 3.81 & 23 \\
\hline & L lingual gyrus & -10 & -89 & 0 & 3.81 & 23 \\
\hline & L declive cerebellum & -10 & -63 & -12 & 3.79 & 22 \\
\hline & R fusiform gyrus & 33 & -37 & -12 & 3.85 & 14 \\
\hline & R fusiform gyrus & 30 & -51 & -9 & 3.79 & 14 \\
\hline & R precuneus & 7 & -54 & 55 & 3.65 & 13 \\
\hline & $\mathrm{L}$ cingulate gyrus & -13 & -37 & 40 & 3.71 & 12 \\
\hline & L lingual gyrus & -13 & -74 & -6 & 3.63 & 11 \\
\hline Photo X amount & L lingual gyrus & -16 & -92 & -3 & -3.50 & 17 \\
\hline
\end{tabular}

$n=22$; voxelwise $p<0.005$ uncorrected, cluster corrected $p<0.05$, minimum cluster size $=103 \times 3 \times 3 \mathrm{~mm}$ contiguous voxels; $x=$ right; $y=$ anterior; $z=$ superior, bold indicates activation of predicted volumes of interest.

visual stream, and fusiform face area), as well as in regions implicated in anticipatory affect (including the NAcc and anterior insula; Table 1, Fig. $3 A, B)$. Low versus high requested amount activated regions implicated in memory and attention (including the parahippocampal gyrus, uncus, precuneus, and left inferior frontal gyrus). In the second model, we predicted that anticipation of giving might also activate regions implicated in anticipatory affect. The contrast of anticipation of giving versus not giving increased activity in the NAcc, along with other regions, but not the anterior insula (Table 2).

\section{Volume of interest activity}

Analyses of activity time courses averaged for photograph versus silhouette trials indicated that photographs significantly increased NAcc activity during concurrent presentation of photographs and amount requested. Photographs also increased anterior insula and amygdala activity during this period as well as during the earlier presentation of photographs versus silhouettes (Fig. 3A). All of these significant increases in activity preceded the choice phase of each trial.

Analyses of activity time courses averaged by the eventual choice to donate or not, however, revealed a more selective pattern. NAcc activity during concurrent presentation of faces or silhouettes and requested amount significantly increased before 
A
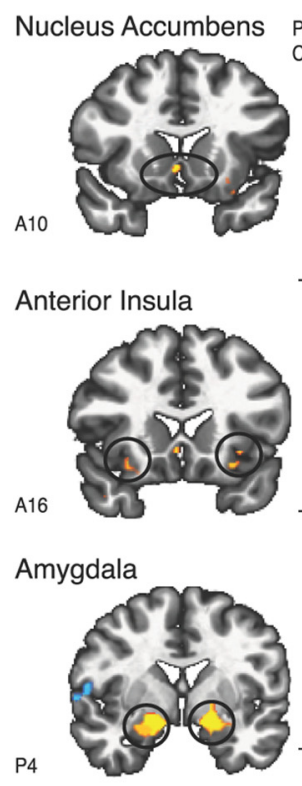

Photo- vs. Silhouette $=-$

$2 \quad \& \$ Y / N$

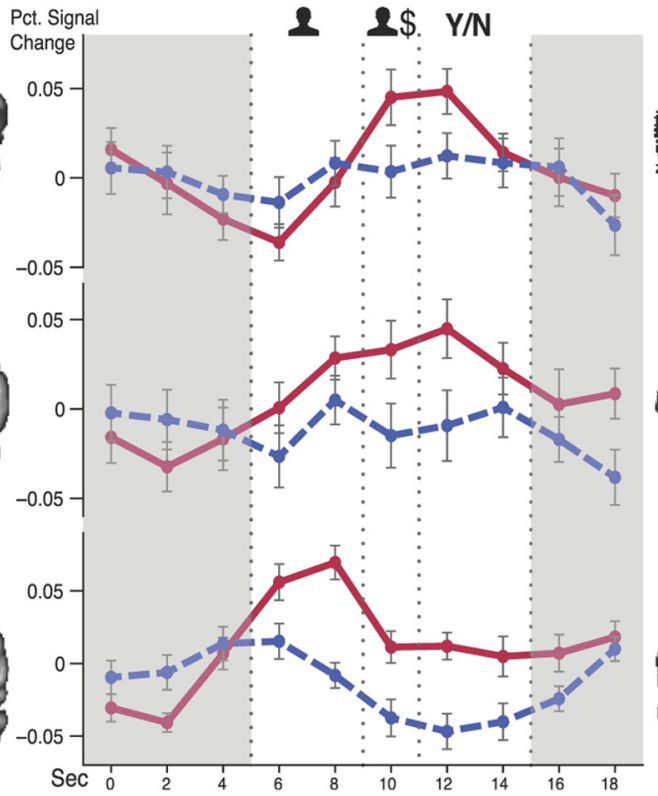

B
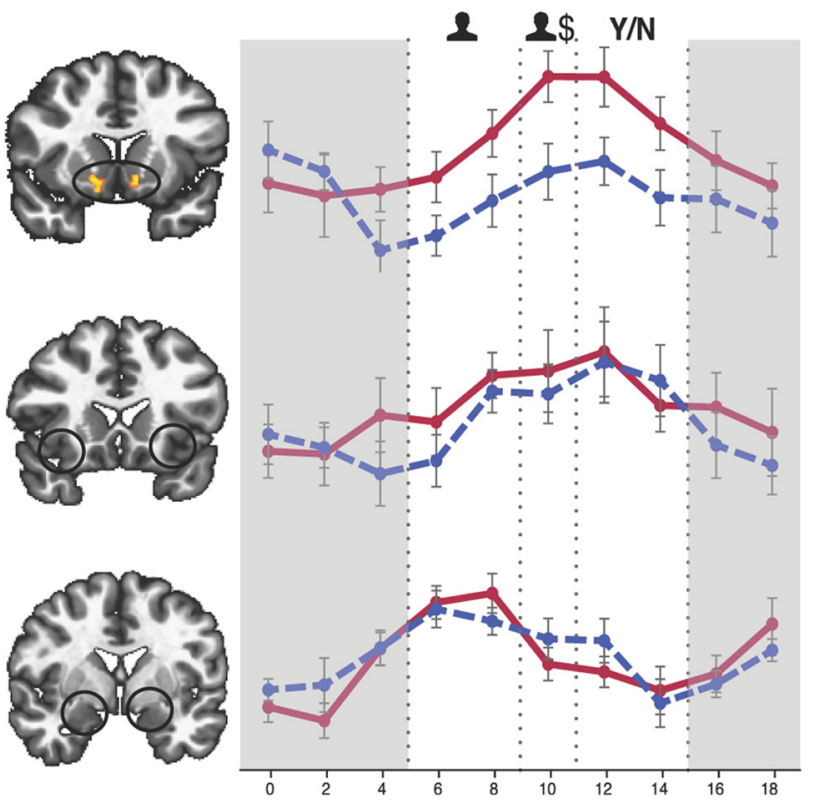

Figure 3. Photograph presentation and anticipation of giving neurally increase NAcc activity. $\boldsymbol{A}$, Group maps $(p<0.05$, corrected) and activity time courses $(x$-axis $=2$ s volume acquisitions; $y$-axis = percentage signal change, error bars $= \pm$ SEM) extracted from NAcc, anterior insula, and amygdala volumes of interest for the Photograph versus Silhouette contrast. $\boldsymbol{B}$, Group maps and activity time courses extracted from NAcc, anterior insula, and amygdala volumes of interest for the Give versus Not Give contrast. Highlighted periods indicate trial phases of interest: onset of photo versus silhouette (figure), requested amount $(\$)$, and choice prompt $(\mathrm{Y} / \mathrm{N})$.

Table 2. Whole-brain analyses contrasting anticipation of giving versus not giving

\begin{tabular}{clrrrrr}
\hline Give versus & L middle occipital gyrus & -25 & -92 & 6 & 4.43 & 299 \\
not give & R parahippocampal gyrus & 24 & -31 & -3 & 3.80 & 66 \\
& R thalamus & 16 & -16 & 3 & 3.72 & 52 \\
& R MPFC & 13 & 41 & 0 & 4.06 & 51 \\
& R fusiform gyrus & 33 & -63 & -6 & 3.79 & 50 \\
& L posterior cingulate & -10 & -48 & 8 & 3.53 & 41 \\
R NAcc & $\mathbf{1 0}$ & $\mathbf{1 0}$ & $-\mathbf{6}$ & $\mathbf{4 . 0 3}$ & $\mathbf{2 8}$ \\
L thalamus & -13 & -16 & 14 & 3.90 & 28 \\
R postcentral gyrus & 39 & -25 & 43 & 3.40 & 23 \\
R superior frontal gyrus & 16 & -27 & 43 & 3.39 & 20 \\
R middle temporal gyrus & -45 & 57 & 11 & 3.43 & 19 \\
L NAcc & $-\mathbf{7}$ & $\mathbf{7}$ & $-\mathbf{9}$ & $\mathbf{4 . 1 1}$ & $\mathbf{1 8}$ \\
L cerebellar dentate & -13 & -60 & -26 & 3.58 & 15 \\
R cerebellar culmen & 30 & -51 & -23 & 3.18 & 15 \\
R uncus & 23 & -2 & -20 & 3.87 & 15 \\
R lingual gyrus & 4 & -66 & 3 & 3.60 & 15 \\
R medial frontal gyrus & 7 & 1 & 49 & 3.56 & 15 \\
R superior temporal gyrus & 62 & -34 & 14 & 4.43 & 14 \\
R precuneus & 19 & -72 & 26 & 3.25 & 14 \\
R anterior cingulate & 4 & 13 & 29 & 3.33 & 12 \\
L parahippocampal gyrus & -28 & -34 & -9 & 3.72 & 11 \\
\hline
\end{tabular}

$n=22$; voxel-wise $p<0.005$ uncorrected, cluster corrected $p<0.05$, minimum cluster size $=103 \times 3 \times 3 \mathrm{~mm}$ contiguous voxels; $x=$ right, $y=$ anterior; $z=$ superior, bold indicates activation of predicted volumes of interest.

subjects chose to donate versus not to donate $\left(t_{(21)}=2.49, p=\right.$ $0.021)$. Anterior insula and amygdala activity, on the other hand, did not significantly differ at any point before subjects chose to donate versus not to donate (Fig. 3B).

\section{Neural prediction of choice}

While presentation of photographs versus silhouettes activated several brain regions implicated in both visual input and anticipatory affect, activity in only a subset of these regions might eventually promote giving. Given the mediating role of positive arousal in the behavioral findings, we suspected that NAcc activ- ity would best predict choices to donate on a trial-to-trial basis. Behavioral, neural, and combined hierarchical logistic regression models were run separately for photograph and silhouette trials (each included subject random effects to control for individual differences). For photograph trials, a first behavioral model indicated that positive arousal predicted increased donations $(Z=$ $2.79, p=0.005)$, whereas negative arousal did not $(Z=-1.64$, $p=0.10$ ). A second neural model (i.e., including NAcc, anterior insula, amygdala, and MPFC activity) indicated that only NAcc activity $(Z=2.50, p=0.013)$ predicted increased donations. Leave-one-subject-out cross-validation of this model for downsampled brain data (i.e., $50 \%$ choices to donate, $50 \%$ choices not to donate) also indicated that predictions significantly generalized across subjects at $56.16 \%\left( \pm \mathrm{SEM}=2.52 \% ; t_{(19)}=2.43 ; p=\right.$ 0.025 , two-tailed). A third combined neurobehavioral model including both behavioral and neural predictors revealed that both positive arousal $(Z=2.87, p=0.004)$ and NAcc activity $(Z=$ 2.99, $p=0.003$ ) continued to predict increased donations (Table $3)$. This combined neurobehavioral model accounted for more variance than the behavioral model, as evidenced by a higher $R^{2}$ ( 0.240 vs 0.224 ), and lower Akaike Information Criterion (AIC; 727.3 vs 729.8 ).

For silhouette trials, the behavioral model indicated that positive arousal predicted increased donations $(Z=3.58, p<0.001)$, while negative arousal instead predicted decreased donations $(Z=-4.46, p<0.001)$. The neural model, however, revealed no significant predictors of donations. The combined neurobehavioral model also indicated that only positive arousal predicted increased donations $(Z=3.55, p<0.001)$, whereas negative arousal instead predicted decreased donations $(Z=-4.49, p<$ 0.001; Table 3). Leave-one-subject-out cross-validation of downsampled brain data indicated that predictions did not significantly generalize across subjects $\left(t_{(19)}=0.54 ; p=0.593\right)$. While the combined neural and behavioral model accounted for slightly 


\begin{tabular}{lccc}
\hline & Behavioral & Neural & Combined \\
\hline $\begin{array}{l}\text { Photograph condition } \\
\text { Positive arousal }\end{array}$ & $2.79^{* *}(0.98,0.34)$ & & $2.87^{* *}(0.98,0.34)$ \\
Negative arousal & $-1.64(-0.67,0.41)$ & & $-1.70(-0.69,0.41)$ \\
NAcc & & & $2.99^{* *}(0.92,0.31)$ \\
Insula & & $2.50^{*}(0.87,0.35)$ & $-0.78(-0.32,0.40)$ \\
Amygdala & & $-0.99(-0.38,0.38)$ & $-0.34(-0.16,0.49)$ \\
MPFC & -320.90 & $-0.40(-0.21,0.52)$ & $1.01(0.19,0.19)$ \\
Log likelihood & 0.224 & $1.31(0.25,0.19)$ & -315.73 \\
$R^{2}$ & 729.8 & -360.25 & 0.240 \\
AlC & & 0.130 & 727.4 \\
Silhouette condition & $3.58^{* * *}(1.83,0.51)$ & 806.1 & $3.55^{* * *}(1.87,0.53)$ \\
Positive arousal & $-4.46^{* * *}(-1.80,0.41)$ & & $-4.49^{* * *}(-1.81,0.40)$ \\
Negative arousal & & & $0.35(0.14,0.39)$ \\
NAcc & & $0.24(0.08,0.33)$ & $-0.78(-0.24,0.31)$ \\
Insula & & $-0.50(-0.17,0.33)$ & $0.73(0.26,0.35)$ \\
Amygdala & -304.0 & $0.91(0.27,0.30)$ & $1.49(0.24,0.17)$ \\
MPFC & 0.259 & $0.93(0.14,0.15)$ & -303.2 \\
Log likelihood & 684.5 & -372.0 & 0.264 \\
$R^{2}$ & 0.097 & 689.4 \\
AIC & & 827.3 & \\
\hline
\end{tabular}

Z-scores with coefficients and SEs in parentheses. Significance: ${ }^{* * *} p<0.001 ;{ }^{* *} p<0.01 ;{ }^{*} p<0.05$. Regressions include subject random effects.

more variance than the behavioral model, a higher AIC indicated that this came at a cost of overfitting the data with additional parameters.

\section{Neural mediation of choice}

Finally, we predicted that trial-based NAcc activity might mediate the influence of photographs on choices to donate. Thus, bootstrapped mediation analysis ( $n=1000)$ were conducted on trial-based data to determine whether NAcc activity could account for the influence of photographs on choices to donate (Fig. 4B). Results revealed both a significant indirect path from photograph to NAcc activity ( $p=0.048, Z=1.98$; coefficient of mediation $=0.017,95 \% \mathrm{CI}=[0.001,0.042])$, and a decreased direct path coefficient from photograph to choices to donate (from 0.87 to $0.57 ; Z=2.05, p<0.040$ ).

When anterior insula, amygdala, or MPFC activity were substituted for NAcc activity in the mediation analysis, paths from brain activity to choice to donate were no longer significant, and the direct path from photograph to choice to donate did not change significantly. Thus, in addition to conditional selfreported positive arousal, trial-based NAcc activity also partially mediated the influence of photographs on choices to donate.

\section{Discussion}

These experiments reveal a neurobehavioral mechanism underlying the identifiable victim effect by demonstrating that affect elicited by identifiable information reliably shifts preferences for giving. Behaviorally, photographs (vs silhouettes) increased donations to orphans by inducing positive aroused affect. Neurally, while photographs (vs silhouettes) increased activity in circuits associated with facial and affective processing, only activity in regions associated with positive arousal (i.e., the NAcc) predicted increased donations. In fact, NAcc activity could statistically account for the photographs' ability to increase giving. While re-
B

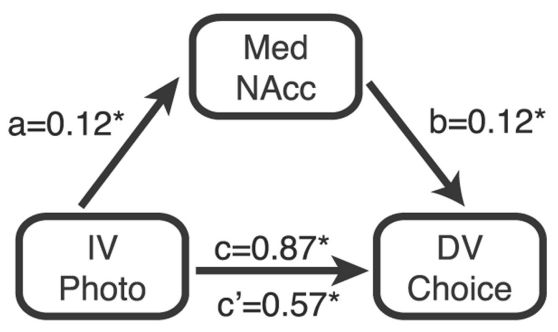

Figure 4. Photographs neurally shift preferences for giving by eliciting NAcc activity. $\boldsymbol{A}$, Percentage donations as a function of requested donation amounts for photograph $(O)$ versus silhouette $(\bigcirc)$ trials. The shift of the fit for photograph trials (solid: mean: $59.4 \%$, SEM: 5.03) versus silhouette trials (dashed: mean: $40.9 \%$, SEM: 4.70 ) to the right indicates greater giving in response to ettes) on decisions to donate. The indirect path from photograph to NAcc activity to donation decision was significant $(p=0.048$, $Z=1.66$; coefficient of mediation $=0.017,95 \% \mathrm{Cl}=[0.001,0.045])$. The direct path coefficient of photograph to donation decision decreased in the model after including the NAcc activation mediator (from 0.87 to $0.57 ; Z=2.05, p<0.040$ ).

searchers have previously argued that affect might influence giving, these findings specifically demonstrate that positive arousal enhances preferences for giving-even within the same individual.

Together, these findings help to distinguish between different accounts of how identifiable information can increase giving. While common intuition and some experimental evidence suggests that negative aroused affect (e.g., guilt) increases giving (Small and Verrochi, 2009; Hein et al., 2010), other evidence implies that positive aroused affect (e.g., an expectant warm glow) encourages giving (Andreoni, 1990; Harbaugh et al., 2007). In the current behavioral study, photograph-elicited positive arousal but not negative arousal increased giving. In a neural extension, NAcc activity, but not anterior insula activity, predicted giving in photograph trials. While both positive arousal and nucleus accumbens activity increased giving, prediction analyses implied partially independent effects. This may be due to the fact that NAcc activity was assessed on each trial immediately before choice, but positive arousal was assessed retrospectively 
for representative trials only, to minimize task disruptions. Surprisingly, in the current experiments, negative arousal decreased rather than increased subsequent decisions to donate. This may be because subjects' negative arousal reacted more to potential costs than identifiable information, consistent with a "pain of paying" account (Prelec and Loewenstein, 1998; Rubaltelli and Agnoli, 2012). Together, these findings suggest that photographs can specifically enhance giving to orphans by inducing positive arousal. Consistent with this account, activity in only a subset of brain regions activated by photographs promoted giving. The lack of predictive activity in face-processing regions extending from the ventral stream of the visual cortex to the amygdala thus implicates affective rather than sensory processes in photographinduced giving (Hsee and Zhang, 2010). While the present conclusions apply to appeals to donate to young orphans, future research will need to test how well this affective mechanism generalizes to other giving scenarios.

The combined neurobehavioral findings thus make several novel contributions to existing research. First, while previous research has demonstrated identifiable victim effects between subjects, these studies provide the first reliable and reversible demonstration of the identifiable victim effect within subjects. Although group effects provide a useful starting point, demonstrations that incidental information can change the same choice within an individual clearly imply inconsistencies in choice that rationality assumptions of individual fixed preferences cannot explain. Within-subjects designs also raise the possibility of investigating how environmental influences can systematically alter preferences, in addition to allowing repeated measurements necessary for neuroimaging research. Second, these findings generally establish a causal role for affect in charitable giving decisions, and specifically suggest a critical role for positive arousal. While previous studies reported affective influences (Bosman and van Winden, 2002; Kogut and Ritov, 2005b) their designs did not allow investigators to test mediation within subjects. Earlier studies were also limited by affect ratings that targeted single task features (e.g., photos alone), rather than subjects' reactions to conjoint decision scenarios (e.g., photos plus monetary requests). Notably, both photograph presentation and the presentation of low requested donations elicited positive arousal, consistent with both empathetic and warm glow accounts of altruistic giving (Batson et al., 1981, 1991; Andreoni, 1990). Third, the predictive neural analyses narrowed the scope of inquiry from a broad range of neural regions whose activity correlated with identifiable features to more focused circuits that promote giving. While many neural circuits preferentially respond to faces (Kanwisher et al., 1997), feelings of empathy (Decety and Jackson, 2006; Lamm et al., 2007), or charitable donations (Moll et al., 2006; Hare et al., 2010), not all of these neural responses critically influence choice (Harbaugh et al., 2007). Indeed, logistic regression analyses of volume of interest data identified the NAcc as the single region responsive to identifiable information whose activity also predicted increased choices to give to orphans depicted with photographs. Further, mediation analyses indicated that NAcc activity could statistically account for the influence of photographs on donations. Together, these findings support the notion that NAcc activity and associated positive arousal can mediate the influence of identifiable information on charitable giving. The ability of photographs versus silhouettes to recruit positive arousal and NAcc activity and thereby to promote giving (even when irrelevant to the task at hand) may describe a specific mechanism underlying more general instances of the identifiable victim ef- fect, which might be more appropriately conceptualized as an "evaluable victim effect."

These findings also highlight a number of promising directions for future research. Better measures of dynamic changes in affect might clarify causes of other economic and social decisions, allowing investigators to determine when affect does and does not contribute to choice. The current design used retrospective affect ratings to avoid priming choice and to minimize taskrelated interruptions during scanning. While these steps ensured that subjects provided the most accurate and unbiased ratings possible, real-time affective probes might potentially improve measurement and better establish mediation (Cooper and Knutson, 2008). Further, many factors have been described as contributing to the identifiable victim effect beyond vivid and salient information. The affective influence of these other forms of identifiable information might vary. For instance, unlike photographs, we found no significant influence of names on the identifiable victim effect, consistent with their lack of an affective impact. Other contextual information, however, including geographic location, local environment, current needs, and individual differences in donors may influence affect and thus alter the identifiable victim effect. Research is needed to determine the impact of each of these factors and whether or not they act through the same affective channels. While the identifiable victim effect may result from presentation of incidental information, it may also result from presentation of individual versus group information (Schelling, 1968). The present findings cannot address whether similar affective mechanisms might also account for statistical variants of the identifiable victim effect, but future research might resolve this important question.

Beyond uncovering basic affective and neural mechanisms that influence giving, this line of research also has practical implications. Improved understanding of the factors that influence charitable donation may facilitate the design of appeals that more effectively promote philanthropic behavior. Charitable organizations continually face the pressing question of whether and how to depict their charges, and knowledge of neurobehavioral mechanisms that promote giving could enhance their strategies. On the other hand, while individuals may understandably make charitable giving decisions based upon the affective impact of potential beneficiaries, these criteria may be less defensible from the utilitarian lens of public policy. Finally, generalizing the findings from charitable giving to broader decisions implies novel directions for future research that focuses on the role of affect in social choice (Lerner et al., 2004; Decety and Jackson, 2006; Hein and Singer, 2008; Zaki and Ochsner, 2012). Donation scenarios provide a unique yet ubiquitous example of how individuals choose to balance interest in the self versus others-even those whom they may never meet or know.

\section{References}

Andreoni J (1990) Impure altruism and donations to public goods: a theory of warm-glow giving. Econ J 100:464-477. CrossRef

Andreoni J (1995) Warm-glow versus cold-prickle: the effects of positive and negative framing on cooperation in experiments. Q J Econ 110:1-21. CrossRef

Andreoni J, Petrie R (2004) Public goods experiments without confidentiality: a glimpse into fund-raising. J Publ Econ 88:1605-1623. CrossRef

Batson CD, Duncan BD, Ackerman P, Buckley T, Birch K (1981) Is empathic emotion a source of altruistic motivation? J Pers Soc Psychol 40: 290-302. CrossRef

Batson CD, Batson JG, Slingsby JK, Harrell KL, Peekna HM, Todd RM (1991) Empathic joy and the empathy-altruism hypothesis. J Pers Soc Psychol 61:413-426. CrossRef Medline 
Bosman R, van Winden F (2002) Emotional hazard in a power-to-take experiment. Econ J 112:147-169. CrossRef

Chang C, Glover GH (2009) Effects of model-based physiological noise correction on default mode network anti-correlations and correlations. Neuroimage 47:1448-1459. CrossRef Medline

Cohen MS (1997) Parametric analysis of fMRI data using linear systems methods. Neuroimage 6:93-103. CrossRef Medline

Cooper JC, Knutson B (2008) Valence and salience contribute to nucleus accumbens activation. Neuroimage 39:538-547. CrossRef Medline

Cox RW (1996) AFNI: software for analysis and visualization of functional magnetic resonance neuroimages. Comput Biomed Res 29:162-173. CrossRef Medline

Decety J, Jackson PL (2006) A social-neuroscience perspective on empathy. Curr Dir Psychol Sci 15:54-58. CrossRef

Fetherstonhaugh D, Slovic P, Johnson SM, Friedrich J (1997) Insensitivity to the value of human life: a study of psychophysical numbing. J Risk Uncert 14:283-300. CrossRef

Grosenick L, Klingenberg B, Katovich K, Knutson B, Taylor JE (2013) Interpretable whole-brain prediction analysis with GraphNet. Neuroimage 72:304-321. CrossRef Medline

Harbaugh WT, Mayr U, Burghart DR (2007) Neural responses to taxation and voluntary giving reveal motives for charitable donations. Science 316:1622-1625. CrossRef Medline

Hare TA, Camerer CF, Knoepfle DT, Rangel A (2010) Value computations in ventral medial prefrontal cortex during charitable decision making incorporate input from regions involved in social cognition. J Neurosci 30:583-590. CrossRef Medline

Hein G, Singer T (2008) I feel how you feel but not always: the empathic brain and its modulation. Curr Opin Neurobiol 18:153-158. CrossRef Medline

Hein G, Silani G, Preuschoff K, Batson CD, Singer T (2010) Neural responses to ingroup and outgroup members' suffering predict individual differences in costly helping. Neuron 68:149-160. CrossRef Medline

Hsee CK, Zhang J (2010) General evaluability theory. Perspect Psychol Sci 5:343-355. CrossRef

Jaeger TF (2008) Categorical data analysis: away from ANOVAs (transformation or not) and towards Logit Mixed Models. J Mem Lang 59:434446. CrossRef Medline

Jenni KE, Loewenstein G (1997) Explaining the "Identifiable Victim Effect." J Risk Uncert 14:235-257. CrossRef

Kanwisher N, McDermott J, Chun MM (1997) The fusiform face area: a module in human extrastriate cortex specialized for face perception. J Neurosci 17:4302-4311. Medline

Knutson B, Greer SM (2008) Anticipatory affect: neural correlates and consequences for choice. Philos Trans R Soc Lond B Biol Sci 363:3771-3786. CrossRef Medline

Knutson B, Taylor J, Kaufman M, Peterson R, Glover G (2005) Distributed neural representation of expected value. J Neurosci 25:4806-4812. CrossRef Medline

Knutson B, Rick S, Wimmer GE, Prelec D, Loewenstein G (2007) Neural predictors of purchases. Neuron 53:147-156. CrossRef Medline
Knutson B, Wimmer GE, Rick S, Hollon NG, Prelec D, Loewenstein G (2008) Neural antecedents of the endowment effect. Neuron 58:814822. CrossRef Medline

Kogut T, Ritov I (2005a) The "identified victim" effect: an identified group, or just a single individual? J Behav Decis Mak 18:157-167. CrossRef

Kogut T, Ritov I (2005b) The singularity effect of identified victims in separate and joint evaluations. Org Behav Hum Decis Mak 97:106-116. CrossRef

Kogut T, Ritov I (2013) The science of giving: experimental approaches to the study of charity. In: The science of giving: experimental approaches to the study of charity (Oppenheimer DM, Olivola CY, eds), pp 133-148. New York: Psychology.

Lamm C, Batson CD, Decety J (2007) The neural substrate of human empathy: effects of perspective-taking and cognitive appraisal. J Cogn Neurosci 19:42-58. CrossRef Medline

Lerner JS, Small DA, Loewenstein G (2004) Heart strings and purse strings: carryover effects of emotions on economic decisions. Psychol Sci 15:337341. CrossRef Medline

Mackinnon DP, Lockwood CM, Williams J (2004) Confidence limits for the indirect effect: distribution of the product and resampling methods. Multivariate Behav Res 39:99. CrossRef Medline

Masten CL, Morelli SA, Eisenberger NI (2011) An fMRI investigation of empathy for "social pain" and subsequent prosocial behavior. Neuroimage 55:381-388. CrossRef Medline

Moll J, Krueger F, Zahn R, Pardini M, de Oliveira-Souza R, Grafman J (2006) Human fronto-mesolimbic networks guide decisions about charitable donation. Proc Natl Acad Sci U S A 103:15623-15628. CrossRef Medline

Preacher KJ, Hayes AF (2008) Asymptotic and resampling strategies for assessing and comparing indirect effects in multiple mediator models. Behav Res Methods 40:879-891. CrossRef Medline

Prelec D, Loewenstein G (1998) The red and the black: mental accounting of savings and debt. Market Sci 17:4-28. CrossRef

Rosen BR, Buckner RL, Dale AM (1998) Event-related functional MRI: past, present, and future. Proc Natl Acad Sci U S A 95:773-780. CrossRef Medline

Rubaltelli E, Agnoli S (2012) The emotional cost of charitable donations. Cogn Emot 26:769-785. CrossRef Medline

Schelling TC (1968) The life you save may be your own. In: Problems in public expenditure analysis. Washington, DC: The Brookings Institute.

Slovic P (2007) If I look at the mass I will never act: psychic numbing and genocide. Judgm Decis Mak 2:79-95.

Small DA, Verrochi NM (2009) The face of need: facial emotion expression on charity advertisements. J Mar Res 46:777-787. CrossRef

Sobel ME (1982) Asymptotic confidence intervals for indirect effects in structural equation models. Sociol Methodol 13:290-312. CrossRef

Watson D, Wiese D, Vaidya J, Tellegen A (1999) The two general activation systems of affect: structural findings, evolutionary considerations, and psychobiological evidence. J Pers Soc Psychol 76:820-838. CrossRef

Zaki J, Ochsner K (2012) The neuroscience of empathy: progress, pitfalls and promise. Nat Neurosci 15:675-680. CrossRef Medline 